\title{
A New Approach to Automatic Signature Complexity Assessment
}

\author{
Oscar Miguel-Hurtado \\ School of Engineering and Digital Arts \\ University of Kent \\ Canterbury UK \\ Email: O.Miguel-Hurtado-98@kent.ac.uk
}

\author{
Richard Guest \\ School of Engineering and Digital Art \\ University of Kent \\ Canterbury UK \\ Email: R.M.Guest@kent.ac.uk
}

Thomas Chatzisterkotis

\author{
School of Engineering and Digital Arts \\ University of Kent, \\ Canterbury UK \\ Email: tc364@kent.ac.uk
}

\begin{abstract}
Understanding signature complexity has been shown to be a crucial facet for both forensic and biometric applications. The signature complexity can be defined as the difficulty that forgers have when imitating the dynamics (constructional aspects) of other users signatures. Knowledge of complexity along with others facets such stability and signature length can lead to more robust and secure automatic signature verification systems. The work presented in this paper investigates the creation of a novel mathematical model for the automatic assessment of the signature complexity, analysing a wider set of dynamic signature features and also incorporating a new layer of detail, investigating the complexity of individual signature strokes. To demonstrate the effectiveness of the model this work will attempt to reproduce the signature complexity assessment made by experienced FDEs on a dataset of 150 signature samples.
\end{abstract}

\section{INTRODUCTION}

Handwritten signatures have long been established as one of the most widely used means of identification at personal, industrial and governmental levels. As examples, most agreements with financial institutions or postal deliveries are followed by the act of signing as a transactional verification. To support the use of signature as transactional verification many end-user companies have adopted electronic write-on screen capture devices in order to incorporate the users signatures into paperless solutions. The paperless solution allows companies to behave in a more eco-friendly manner and, at the same time, reduce cost and improve work flow processes. Furthermore it has been shown that there is high customer acceptability in the use of signature using write-on devices [1]. These technologies have renewed interest in dynamic automatic signature verification (AVS) systems wherein both the static (outcome) and dynamics (constructional/temporal) elements are considered in comparison. Current AVS systems have achieved very promising error rates and are considered as a mature biometric modality [2]. A number of recent studies have assessed usability aspects of ASV systems wherein not only the algorithmic performance was analysed, but also how the users interact with the technology [1].

The handwritten signature is a behavioural biometrics modality, and therefore is susceptible to fraudulent attempts by imitating the sequence of movement that create the signature output. Within the forensic document examiners (FDE) community it is accepted that there are a range of signatures, some easier to imitate than others. Found and Rogers [3] define signature complexity as the difficulty that forgers have when imitating the dynamics (constructional aspects) of other users signature. This degree of difficulty supports FDE in their decisions whether a signature sample is genuine or an imitation. As a biometric means of authentication, a signature can be thought of as being analogous to conventional passwords in terms of definition and use, with three aspects to which a robust signature should comply:

a) Quantity of data: this can be akin to the length of a password. Most common password-based authentication systems force users to choose a minimum number of characters for their passwords. In a dynamic signature, this can be translated as ensuring enough constructional sample points which relates to the temporal length of a signatures construction.

b) Consistency of the signature: as a behavioural biometrics, dynamic signature have an inherent variability. ASV systems are designed to manage this variability, generally through tolerance within pattern recognition techniques. However, this variability cannot be excessively large, otherwise it could compromise the security of the solution. Applying the password analogy, users are expected to use the same password at each attempt. In the same manner, users of ASV systems should provide the same signature each time (within an acceptable degree of variability).

c) Complexity of the signature: as previously stated, the complexity of a signature can be described as the difficulty of being imitated. Within the password analogy this can be interpreted as the rules which reject simple passwords and force users to incorporate upper and lower case characters and/or the inclusion of numbers. In the signature domain, complexity has been linked to the number of changes in direction (also known as singular points) and the number of intersections between the signature lines [4].

Consequently, there is a consensus that knowledge of signature complexity can help the ASV systems to improve their performance. As in other biometric modalities, the quality of the input sample has an effect on the performance of biometric systems. A clear example of this is the NFIQ (NIST Fingerprint Image Quality) used in fingerprint biometric 
systems [5]. The signature complexity can be used as a quality check for ASV systems, therefore requiring a method to automatically analyse the complexity of signatures after collection. Complexity can be used within ASV systems to reject very simple signatures that do not comply with minimum security requirements. Signature complexity levels can also be used to tune comparison engines with different settings at algorithmic level providing the potential to allow an enhanced and/or more secure method to compare simple or complex signatures.

Inspired by the work of Found and Rogers [4], the authors have revisited the task of automatic signature complexity assessment from a biometric perspective. Using the dynamic data available from the signature capture devices, FDE static signature complexity features can be automatically extracted and complemented with dynamic signature features to improve the complexity assessment. The aim of this work therefore is to investigate on the creation of a mathematical model for the automatic assessment of the signature complexity. We explore the effect of increasing the number of features analysed and also incorporate a new layer of detail, investigating the complexity of individual signature strokes. To demonstrate the effectiveness of the model this work will attempt to reproduce the signature complexity assessment made by experienced FDEs.

\section{LITERATURE REVIEW}

The need for objective measurements in FDE was the primary motive for the development of a complexity theory. Huber stated in his work that "the complexity of writing movement is thought to be critical for the reliability of the examination process" [6]. Signature complexity theory is based therefore on two basic principles: i) the more material there is to for the comparison of a disputed signature of a person the easier it is for the expert to reach safer conclusions, ii) the more complex the writing of a person, the more difficult it is to be copied by another individual [7].

A state-of-the-art article for ASV stated that a common problem for systems is caused by the complexity of sample signatures [2]. When a signature is small without many features and often similar characteristics, these carry less information with a higher likelihood that the system will produce the wrong outcome.

Several researchers have discussed the complexity theory with respect to a person to executing different types of handwritten tasks. In a study by Wing [8], a relationship was found between the reaction time (the preparation time that is needed by an individual and the required mental effort to execute a task) and the complexity of writing letters of the alphabet. Subsequently Hong also observed an effect between the pressure and complexity, the pen pressure performed by the participants when were asked to write on a writing surface was higher in more complicated writing tasks [9].

Found and Rogers [4], [10], [11] proposed a complexity theory which is based on the fact that as the complexity of a signature increases, the likelihood of the potential for a correct FDE opinion increases. Using a discriminant function analysis, the best static predictors appeared to be the number of turning points and the number of intersections. A statistical model with 3 equations were proposed to classify the signatures on a three-point complexity scale based on these objective predictors [4], where $72.9 \%$ of the complexity scores by 14 FHEs were predicted correctly by the model. Dewhurst et al. [12] in another study found that the opinions of specialists varied, however the statistical model managed still to correctly classify $75 \%$ of signatures based on a consensus of responses.

Within the biometric community several studies have analysed the impact of signature complexity on the verification algorithms performance. In 1993, Brault and Plamandon analysed this impact in [13], validating than shorter signatures convey generally less information than longer ones, and therefore, achieve lower verification performances. Following this idea, Fairhurst et al. [14] investigated several issues relating to signature complexity and authentication decisions, showing the impact of signature complexity levels in the error rates obtained and acknowledging the need of further investigation on signature complexity methods. In 2007, Alonso-Fernandez et al. [15] analysed the impact of legibility on signature verification performance concluding that the most complex flourish signatures are more robust to skilled forgeries.

\section{Methodology}

In this section our methodology to find the best set of features than can model signature complexity assessment will be detailed. Firstly, the capture protocol and demographic information of the signature database used in our analysis will be provided. Samples from this database were processed to create an assessment form which was sent to the FDEs in order to obtain their expert signature complexity assessments. Furthermore we will describe the dynamic signature features analysed in this work, the feature selection technique performed and the statistical model created to replicate FDE outputs.

\section{A. Database}

The signature database used in this study is part of a wider collection by the University of Kent made in 2008 [16]. It comprises 150 participants and contains both signature samples and handwriting text. Participants donated the signature data both within a constrained signing box (of $80 \mathrm{~mm}$ by $30 \mathrm{~mm}$ ) and without constraints. The signature data was captured using a Wacom Intuos 2 tablet. This device captured the movement of the pen with a sampling rate of $100 \mathrm{~Hz}$, providing the $\mathrm{X}$ and $\mathrm{Y}$ coordinates of the pen, the pen-tip pressure exerted and two angles to spatially locate the pen: the azimuth and the altitude. The database cover a wide range of ages and a representative sample of gender, handedness and writing language. Table I specifies the percentage of participants on each category:

\section{B. Forensic document examiners assessment}

Using the signatures without constraints from the Kent database, static images were sent to three leading professional 
TABLE I: Signature database participant distributions

\begin{tabular}{|c|c|r|}
\hline \multirow{4}{*}{ Age group } & $18-29$ & $55 \%$ \\
\cline { 2 - 3 } & $30-40$ & $10.50 \%$ \\
\cline { 2 - 3 } & $40-50$ & $6 \%$ \\
\cline { 2 - 3 } & $50-60$ & $10.50 \%$ \\
\cline { 2 - 3 } & $60-70$ & $11.30 \%$ \\
\cline { 2 - 3 } & Over 70 & $6.70 \%$ \\
\hline \multirow{4}{*}{ Gender } & Male & $39.90 \%$ \\
\cline { 2 - 3 } & Female & $60.10 \%$ \\
\hline \multirow{3}{*}{ Handedness } & Right & $91 \%$ \\
\cline { 2 - 3 } & Left & $9 \%$ \\
\cline { 2 - 3 } & English & $81 \%$ \\
\hline Writing language & Western & $8 \%$ \\
\cline { 2 - 3 } & Non-western & $11 \%$ \\
\hline
\end{tabular}

forensic document examiners in the UK. These forensic document examiners have considerable expertise over many years through a wide variety of investigations and cases at different national and international courts. 150 signature sample images were arranged on a form divided into eight signatures per page reproduced at normal size. Below each signature were three options for complexity assessment: High, Medium or Low. Each FDE analysed the signatures independently drawing on their individual expertise and experience. In addition, at the end of the document, the FDE were also asked to describe briefly the major factors that led them to select one of the three signature complexity level. Figure 1 shows the signature complexity distribution of the opinion provided by the three FDEs.

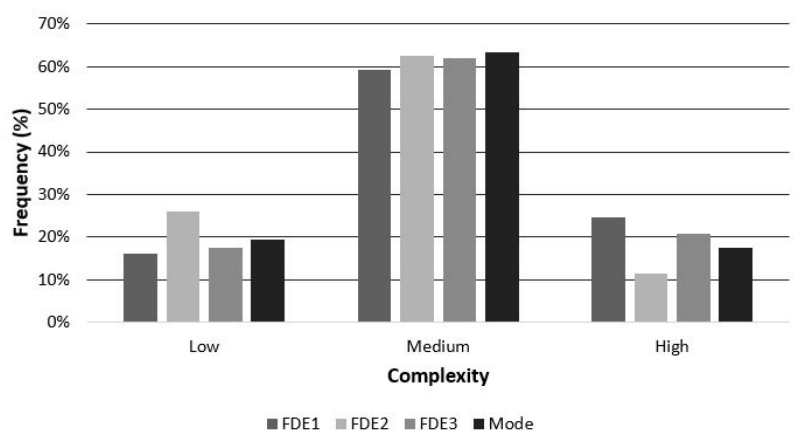

Fig. 1: Signature complexity histograms from the three FDE (and the modal response across the FDEs)

The three FDEs agreed on 93 of the signatures (62\%) whilst in the remaining 57 signatures, at least two of them agree. Within these 57 signatures, in 28 cases there was a disagreement between assigning low or medium complexity. In other 29 cases the disagreement was between considering medium or high complex. In Figure 1, the signature complexity level mode (the most accepted value) is also represented (black column). This modal response was selected as the representative complexity value in order to create and evaluate the signature complexity models.

The main factors indicated by the FDEs when assigning high signature complexity was: i) the existence of multiple pen strokes and whether they overlap or not, ii) the existence of multiple changes in directions, iii) length, iv) the difficult to determine the path of strokes sequence followed by the signers and $v$ ) the degree of signature illegibility. If a signature was short with a simple structure and clean path it was considered of low complexity (see Figure 2 for high and low complexity signature examples). The signature which werent considered low or high complex would fall consequently in the medium complexity level. FDEs based their assessment mainly on static features extracted from the signature image. A number of techniques allow FDEs to extract or estimate dynamic information such pressure or velocity. However, due to the time required for the task of assessing 150 complexity signatures, these techniques were not applied by the FDEs. In this work, the use of automated extraction of dynamic information such as pen velocity or acceleration will enable the systematic and accurate location of points such as intersections, changes of writing direction or stroke detection adding new layers of information for use in the complexity assessment.
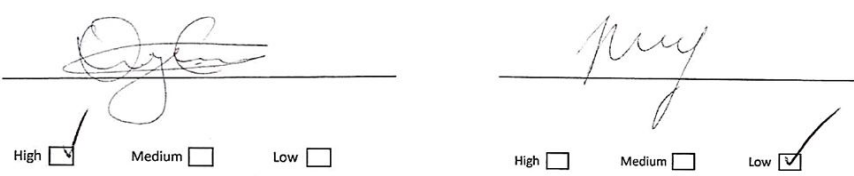

Fig. 2: Signature samples of high and low signature complexity

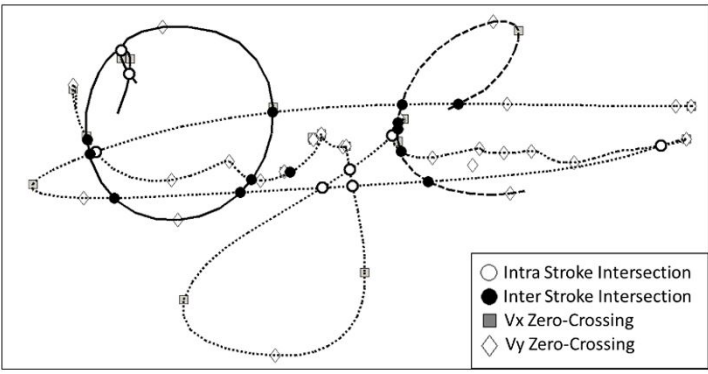

Fig. 3: Signature image with Inter and intra stroke intersection points and Zero-crossing points for $\mathrm{X}$ and $\mathrm{Y}$ velocity

\section{Signature feature extraction}

Based on the literature review and the FDE indications, the following set of static and dynamic features were extracted from each signature to enable a signature complexity assessment:

1) Number of strokes: the number of pen-down events within a full signature. In Figure 3, the strokes are represented by line styles: solid, dashed and dotted.

2) Total number of intra-stroke intersections: this feature denotes the sum of all the intersections points from every individual stroke from a signature. Example of intrastroke intersections can be seen at Figure 3, marked with white circles.

3) Total number of inter-stroke intersections: this feature denotes the sum of all the intersections point across different strokes from a signature. Example of inter-stroke 
intersections can be seen at Figure 3, marked with black circles.

4) Mean of intra-stroke intersections: the average number of intra-stroke intersections amongst the different strokes of a signature.

5) Maximum of intra-stroke intersections: the maximum number of intra-stroke intersections amongst the different strokes of a signature.

6) Mean of inter-stroke intersections: the average number of inter-stroke intersections amongst the different strokes of a signature.

7) Maximum of inter-stroke intersections: the maximum number of inter-stroke intersections amongst the different strokes of a signature.

8) Number of $\mathbf{X}$-axis intersections: As UK population generally writes from left to right, the X-coordinate generally shows a tendency to increase across signing time. This effect can be seen in the X-coordinate vs. time graph as a linear component of a regression line. This feature denotes how many times the X-coordinate signal intersect an imaginary line that joins the start and the end points. The value is an indication of how many times the pen moved backwards (to the left). Examples of these crossing can be seen at the first graph of Figure 4, where X-coordinate is represented by the black line, and the imaginary line from the start to the end sample points is represented in grey. The intersections are marked with white diamonds.

9) Total number of Vx zero-crossings: this feature denotes how many times the $\mathrm{X}$-coordinate change direction from increasing to decreasing or vice-versa. In order to find these changes the first time derivative, the velocity of $\mathrm{X}$ coordinate $(\mathrm{Vx})$, is calculated and the number of time it cross the zero line (denoting a change from increasing to decreasing or vice-versa) value is found. In Figure 4, the $\mathrm{X}$-coordinate velocity is represented, along with the zerovelocity line and examples of the zero-crossing points marked with white diamonds.

10) Total number of Vy zero-crossings: as in feature 9, but using the Y-coordinate.

11) Total number of Ax zero-crossings: in order to add another level of complexity measurement, the changes in pen velocity, from increasing to decreasing and viceversa, have been analysed. The temporal derivative of the velocity, the acceleration in $\mathrm{x}$-coordinate $(\mathrm{Ax})$, has been calculated. This feature denotes the total number of zero-crossing-point found in Ax. In Figure 4, the $\mathrm{x}$ axis acceleration is represented, along with the zeroacceleration line and examples of the zero-crossing points marked with white diamonds.

12) Total number of Ay zero-crossings: as in feature 11, but with the Y-coordinate.

13) Signature length: the signature length has been calculated as the sum of the Euclidean distances between sample points.

14) Normalized signature length: in order to obtain a robust value which is not dependant on the size of the signature, the signature length is normalized by the diagonal length of the signature minimum bounding box.

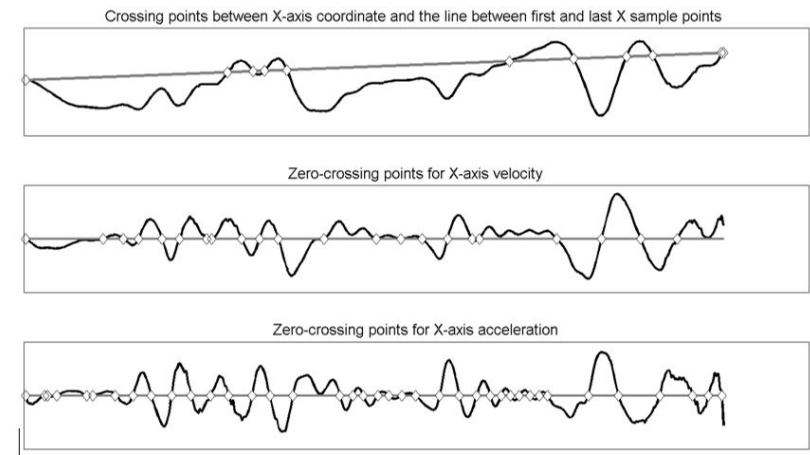

Fig. 4: Crossing points for X-Coordinate

\section{Statistical model and feature selection}

Following the extraction of these 14 features from each signature, the power to predict the complexity of signature was analysed using statistical models. Using, as a response of the model, the complexity indicated by the FDEs (which was numerically translated to: 1 for Low, 2 for Medium and 3 for High complexity) multi-linear regression models have been created for different combinations of features. Multilinear regression models represent the relationship between the explanatory variables (the different combination of the 14 features extracted) and the response variable, the signature complexity, by fitting a linear equation to the observed data. The linear equation has the following form:

$$
y=c_{0}+\sum_{i=1}^{n}\left(c_{i} \cdot x_{i}\right)
$$

where $y$ is the response variables (in our case the signature complexity), $x_{i}$ is the $\mathrm{i}$-th feature extracted, $c_{i}$ is the $\mathrm{i}$ th regression coefficient for that specific feature, $c_{0}$ is the intercept of the linear regression model and $n$ is the number of features included in the model. In order to find the optimum set of features to predict the signature complexity, an exhaustive search was implemented. As the search space is made out of 14 potential features, it represents $2^{4}-1(=16383)$ possible combinations.

\section{RESULTS}

The 14 signature features were calculated for all the 150 signers within the dataset. The mean and standard deviation of feature results can be analysed when grouped by the complexity level assigned by the three FDEs. Table II shows the values obtained for each feature.

It is possible to note how all the features have a higher mean value as the complexity level increase. However, the standard deviations for each feature also increase, especially for the high signature complexity levels. For the modelling stage, in order to obtain coefficient values more easily comparable, feature values have been Z-normalized. Z-normalization 
TABLE II: Feature value mean and standard deviation grouped by signature complexity level

\begin{tabular}{|c|c|c|c|c|c|c|}
\hline Feature & \multicolumn{2}{|c|}{ Low } & \multicolumn{2}{|c|}{ Medium } & \multicolumn{2}{|c|}{ High } \\
\hline 1 & 4.10 & $(2.35)$ & 5.26 & $(3.65)$ & 5.62 & (4.09) \\
\hline 2 & 6.93 & $(4.53)$ & 12.69 & $(8.72)$ & 25.15 & (18.41) \\
\hline 3 & 5.90 & $(4.48)$ & 10.13 & (7.07) & 16.54 & (14.41) \\
\hline 4 & 1.47 & $(3.16)$ & 2.58 & (6.66) & 7.15 & $(15.00)$ \\
\hline 5 & 1.90 & $(3.06)$ & 3.61 & $(6.61)$ & 10.73 & (14.76) \\
\hline 6 & 2.46 & $(2.90)$ & 4.32 & $(6.33)$ & 8.43 & $(14.57)$ \\
\hline 7 & 4.59 & (3.49) & 7.04 & $(6.31)$ & 11.69 & (14.17) \\
\hline 8 & 6.72 & $(3.73)$ & 9.27 & $(5.59)$ & 8.65 & $(8.17)$ \\
\hline 9 & 25.76 & (12.09) & 37.40 & (16.07) & 46.77 & (23.24) \\
\hline 10 & 26.83 & (12.54) & 39.98 & $(16.20)$ & 48.69 & (20.60) \\
\hline 11 & 34.31 & (15.91) & 49.92 & (21.44) & 61.77 & $(28.86)$ \\
\hline 12 & 35.10 & (16.84) & 48.82 & (21.81) & 60.54 & (28.60) \\
\hline 13 & 16.16 & $(5.46)$ & 24.04 & $(7.86)$ & 37.59 & (14.31) \\
\hline 14 & 3.10 & $(0.91)$ & 3.72 & (1.15) & 5.21 & (1.34) \\
\hline
\end{tabular}

transforms a variable to have 0 mean and 1 standard deviation value. This is made by subtraction of the mean variable value and dividing by its standard deviation. It is worthy to remind that z-normalization does not affect the fit of the model, only made a change on the coefficient values. Both model, with and without z-normalization will have the same r-square values and error distributions. Furthermore both models will provide the same signature complexity decision. Based on the accuracy to replicate the signature complexity decisions made by the three FDEs, the best model obtained contained the following feature set (Table III):

TABLE III: Coefficient values for the signature complexity model based on the mode value

\begin{tabular}{lc}
\multicolumn{1}{c}{ Feature } & Coefficient \\
\hline Intercept & 1.98 \\
Number of strokes & -0.09 \\
Number of intra-stroke Intersections & -0.12 \\
Mean of inter-strokes intersection & -0.99 \\
Maximum of inter-stroke intersections & 0.65 \\
Mean of intra-stroke intersections & 0.53 \\
Total number of Vy zero-crossing & 0.38 \\
Total number of Ax zero-crossings & 0.09 \\
Total number of Ay zero-crossings & -0.30 \\
Length & 0.25 \\
\hline
\end{tabular}

This model shows a root mean squared error (RMSE) of 0.457 with an R-squared value of 0.468 (the predictors explain $47 \%$ of the response variation). It is noticeable that there are negative values of the coefficients for the number of strokes, the number of intra-strokes intersections and the mean of inter-strokes intersection. The reason behind these negative values is to moderate the importance of these variables in the calculation prediction of signature complexity. A greater number of strokes does not necessarily imply a higher signature complexity. For example, the addition of several simple and short strokes would generally decrease the signature complexity. On the contrary, the addition of highly complex strokes (with great number of intersection and changes in both directions) would result in a rise the overall signature complexity. In a similar way, the negative value of the total number of Ay zero-crossing may compensate the importance of the total number of Vy zero-crossing. Figure 5 shows a graphic representation of the model, where y-axis represent the output of the model and $\mathrm{x}$-axis represent a combination of feature input values made just for this representation.

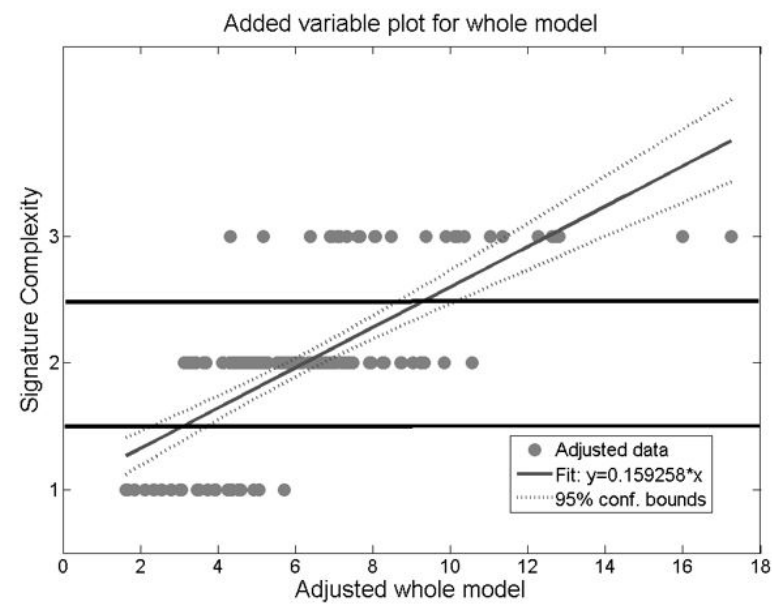

Fig. 5: Added variable plot for signature complexity

Using this model, we can categorize the signature complexity levels by using complexity level thresholds. In this study we apply a high complexity threshold of 2.5 and low complexity threshold of 1.5. These thresholds are represented as black horizontal lines in Figure 5. Using these thresholds signature complexity has been categorized as:

a) Low when: signature complexity $<1.5$

b) Medium when: $1.5<=$ signature complexity $<=2.5$

c) High when: signature complexity $<2.5$

Comparing the complexity levels obtained after applying these thresholds with the complexity levels assessed by each FDE and also with the modal prediction for each signature, we obtain the following results, Figure 6:

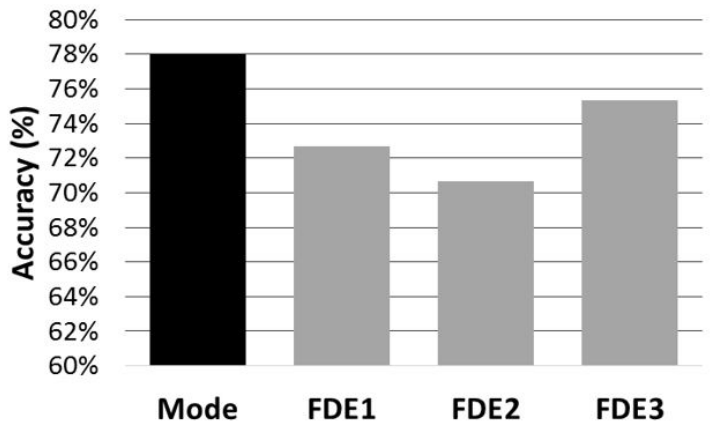

Fig. 6: Accuracy obtained based on the mode complexity level and the individual FDEs levels

As expected, the model created using the modal responses has the highest accuracy achieving a 78\% success rate, which represent an improvement over the results presented at the literature review section. If we compare the outputs with the individual prediction from each FDE, Figure 6 shows that the 
FDE3 obtains $75 \%$ while the FDE1 and 2 obtain $73 \%$ and $71 \%$ respectively. FDE2 presented the most noticeable difference for signature complexity (see Figure 1). The FDE2 produced a higher frequency of low complexity signature than the other FDEs, and a lower frequency for high complexity signature. This explains the low accuracy of the model when applied to the complexity levels assessed by this FDE.

Finally, using the same input features previously selected, we created a new multi-linear model, not using the modal signature complexity levels from the three FDEs, but using the mean value. Figure 7 shows the added variable plot for this model:

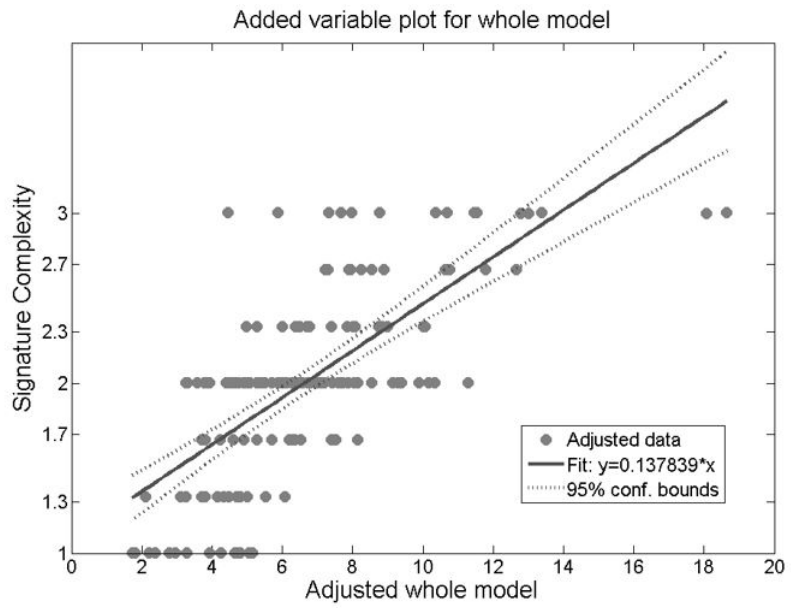

Fig. 7: Added variable plot for the multi-linear regression model created with the mean complexity values

This new model has an RMSE value of 0.406 , with and $\mathrm{R}$-squared of 0.492 . This values represent a better fit of the data than previous model, which is explained by the higher granularity of the signature complexity levels used. In Table IV, the coefficient values for this model are detailed:

TABLE IV: Coefficient values for the signature complexity model based on the mean value

\begin{tabular}{lc}
\multicolumn{1}{c}{ Feature } & Coefficient \\
\hline Intercept & 1.99 \\
Number of strokes & -0.09 \\
Number of intra-stroke Intersections & -0.09 \\
Mean of inter-strokes intersection & -0.85 \\
Maximum of inter-stroke intersections & 0.53 \\
Mean of intra-stroke intersections & 0.50 \\
Total number of Vy zero-crossing & 0.36 \\
Total number of Ax zero-crossings & 0.12 \\
Total number of Ay zero-crossings & -0.33 \\
Length & 0.23 \\
\hline
\end{tabular}

\section{CONCLUSSIONS AND FUTURE WORK}

In this work we have revisited the idea of automatic assessment of signature complexity. With a signature database of 150 samples and the collaboration of professional FDEs, a new set of signature features has been analysed. As can be seen in the complexity assessment of the FDEs, the human inspection still implies a subjective categorization of signature complexity, revealed in the $32 \%$ of signatures where there is disagreement between assessors.

A new set of signature complexity features has been analysed. This set includes some of the more used features found in the ASV literature (as length, time and overall singular points) but also incorporate a more detailed feature level, taking into consideration the complexity structure of individual strokes.

As a result of the analysis, a multi-linear regression model has been obtained. This signature complexity model has been able to replicate the FDEs evaluation with an accuracy of 78\%, providing a more objective way to assess signature complexity. The features found within this model are a combination of common features such as number of strokes and number of changes in velocity (y-axes) with new stroke-detail features such the number of intra-stroke intersections or the mean of inter-strokes intersection. Furthermore, the inclusion of zerocrossing acceleration points also have been found to increase the overall accuracy of the model.

These new features could add a new layer within the automated signature complexity theory. Treating the signature as a sequence of strokes, each of them with its individual complexity led to a more reliable assessment of the overall signature complexity.

As a future work, the authors plan to expand this idea and create a new stroke complexity index. Instead of assessing the signature complexity as a whole, we will assess the complexity of every individual stroke and analyse different ways to create from these individual complexity levels an overall signature complexity level. This stroke complexity index could be used to create new models for signature complexity and improve even more the objectivity and accuracy of the automatic signature complexity assessment systems.

A reliable signature complexity model will benefit both forensics and biometrics state of the art. The FDE community will have available new tools to help their decision on the authenticity of a signature. Furthermore, the biometric community will benefit from the automatic assessment of signature complexity by incorporating it to the actual deployed ASV systems in order to improve their performance and security levels.

\section{ACKNOWLEDGMENTS}

The authors gratefully acknowledge the contribution made by our professional FDE colleagues for their work analysing the signature samples.

\section{REFERENCES}

[1] R. Blanco-Gonzalo, O. Miguel-Hurtado, R. Sanchez-Reillo, and A. Gonzalez-Ramirez, "Usability analysis of a handwritten signature recognition system applied to mobile scenarios," in Security Technology (ICCST), 2013 47th International Carnahan Conference on, 2013, pp. $1-6$.

[2] R. Plamondon, G. Pirlo, and D. Impedovo, "Online Signature Verification," in Handbook of Document Image Processing and Recognition, D. Doermann and K. Tombre, Eds. Springer London, 2014, pp. 917 947. 
[3] B. Found and D. Rodgers, "A consideration of the theoretical basis of forensic handwriting examination," International Journal of Forensic Document Examiners, vol. 4, pp. 109-118, 1998.

[4] B. Found, D. Rogers, V. Rowe, and D. Dick, "Statistical modelling of experts' perceptions of the ease of signature simulation," Journal of Forensic Document Examination, vol. 11, pp. 73-99, 1998.

[5] E. Tabassi and P. Grother, Fingerprint image quality. Springer US, 2009.

[6] R. A. Huber and A. M. Headrick, Handwriting identification: facts and fundamentals. CRC Press, 1999.

[7] B. Found, "Handwriting and Signatures, Comparison of," in Wiley Encyclopedia of Forensic Science. John Wiley \& Sons, Ltd, 2009.

[8] A. M. Wing, "Response timing in handwriting," Information processing in motor control and learning, pp. 153-172, 1978.

[9] M. P. Hong, "Handwriting pressure: Effects of task complexity, control mode and orthographic difference," Graphonomics: Contemporary research in handwriting, Elsevier, North-Holland, pp. 47-66, 1986.

[10] B. Found, D. Dick, and D. Rogers, "The structure of forensic handwriting and signature comparisons," International Journal of Speech Language and the Law, vol. 1, no. 2, pp. 183-196, 1994.

[11] B. Found and D. Rogers, "The forensic investigation of signature complexity," Handwriting and Drawing Research: Basic and Applied Issues, pp. 483-492, 1996.

[12] T. N. Dewhurst, B. J. Found, and D. K. Rogers, "The relationship between quantitatively modelled signature complexity levels and forensic document examiners' qualitative opinions on casework." Journal of Forensic Document Examination, vol. 18, pp. 21-40, 2007.

[13] J. Brault and R. Plamondon, "A complexity measure of handwritten curves: modeling of dynamic signature forgery," Systems, Man and Cybernetics, IEEE Transactions on, vol. 23, no. 2, pp. 400-413, 1993.

[14] M. Fairhurst, E. Kaplani, and R. Guest, "Complexity measures in handwritten signature verification," in Human-Computer Interaction, 2001, pp. 305-309.

[15] F. Alonso-Fernandez, M. Fairhurst, J. Fierrez, and J. Ortega-Garcia, "Impact of Signature Legibility and Signature Type in Off-Line Signature Verification," in Biometrics Symposium, 2007, 2007, pp. 1-6.

[16] J. Chapran, M. Fairhurst, R. Guest, and C. Ujam, "Task-related population characteristics in handwriting analysis," IET Computer Vision, vol. 2, no. 2, pp. 75-87(12), Jun. 2008. [Online]. Available: http://digital-library.theiet.org/content/journals/10.1049/ietcvi_20070069 